\title{
Age and Bone Mass as Predictors of Fracture in a Prospective Study
}

Siu L. Hui, Charles W. Slemenda, and C. Conrad Johnston, Jr. Department of Medicine, Indiana University School of Medicine, Indianapolis, Indiana 46202; and the Regenstrief Institute, Indianapolis, Indiana 46202

\begin{abstract}
To study the effect of bone mass on the risk of fracture, we followed 521 Caucasian women over an average of $6.5 \mathrm{yr}$ and took repeated bone mass measurements at the radius. We observed 138 nonspinal fractures in 3,388 person-yr. The person-years of follow-up and the incident fractures were crossclassified by age and bone mass. The incidence of fracture was then fitted to a log-linear model in age and bone mass. It was found that incidence of fracture increased with both increasing age and decreasing radius bone mass. When subsets of fractures were examined it was found that age was a stronger predictor of hip fractures, whereas midshaft radius bone mass was a stronger predictor of fractures at the distal forearm. We concluded that bone mass is a useful predictor of fractures but that other age-related factors associated with fractures need to be identified.
\end{abstract}

\section{Introduction}

Osteoporosis is defined as a condition of low bone mass with increased susceptibility to fractures. However, available evidence is not very strong in showing a direct relationship between the risk of fracture and the level of preexisting bone mass.

The strongest evidence comes from in vitro experiments demonstrating a direct relationship between bone mass and bone strength. It has been shown that $\sim 80-90 \%$ of bone strength can be explained by its mass (1). Various studies on human subjects in vivo, however, have shown a weaker association. Although most studies have shown that subjects with fractures have significantly lower mean bone mass than controls of similar age, the overlap between the two groups is so large that the bone-mass measurement is of little value in discriminating between individuals with and without fracture (2, 3). Furthermore, most of these studies have been performed retrospectively. When bone mass is measured after the fractures, bone loss as a result of, rather than as a cause of, fracture cannot be excluded. The few prospective studies that show increased risk of fracture with lower bone mass $(4,5)$ are based on small numbers of fractures and have some methodologic problems, primarily the failure to control for age in the analyses.

Address all correspondence to Dr. Siu L. Hui, Regenstrief Health Center, Fifth Floor, 1001 West Tenth Street, Indianapolis, IN 46202.

Received for publication 14 October 1987 and in revised form 28 December 1987.

J. Clin. Invest.

(c) The American Society for Clinical Investigation, Inc. 0021-9738/88/06/1804/06 $\$ 2.00$

Volume 81, June 1988, 1804-1809
In this article, we present a prospective study on more than 500 subjects with repeated bone-mass measurements over 15 yr. This allows for the first time the estimation of the risk of fracture as a simultaneous function of age and the changing bone mass in the individual.

\section{Methods}

Study subjects. The subjects $(n=521)$ were volunteers recruited for a study on the natural history of bone loss in Caucasian women. The original cohort was formed in 1971 but new subjects have been continuously added over the following $15 \mathrm{yr}$. The younger subjects were drawn primarily from gynecologic patients and workers at the Indiana University Medical Center, their friends, and residents in Franklin, a small town about $30 \mathrm{mi}$ from Indianapolis. Many of the older subjects ( $\sim 80 \%$ of those over age 65 ) were ambulatory residents of a retirement home in Franklin. Subjects were excluded if they had life-threatening illnesses, if they were unable to give informed consent, or if they had conditions thought to affect mineral metabolism or bone. Subjects taking medications known to affect mineral metabolism (e.g., corticosteroids, anticonvulsants, and estrogens) were also excluded. All subjects made one to four visits per year for bone-mass measurements, a brief clinical review, and a history of recent fracture. All subjects included in the present study must have had two or more measurements of bone mass over more than $1 \mathrm{yr}$; the average was 24.6 bone-mass measurements over $6.5 \mathrm{yr}$. This research was approved by the Indiana University Institutional Review Board, and all subjects provided informed consent.

Bone mass measurement. The bone width and mass of each subject was measured at the midshaft of the radius using Norland single photon absorptiometers with long-term reproducibility (based on the SD about the regression line for individual bone-mass overtime) of $\sim 2 \%$, as has been previously published (6).

Documentation of fractures. A history of fractures was taken at each visit. Fractures before the first bone-mass measurement were not included in this prospective study. In subjects residing at the retirement home, a technician verified the site of fracture from $x$-ray reports. Subjects residing at home were asked whether the fracture had been diagnosed by a physician and only those fractures that were reported to be seen on $x$-ray were included. Since only fractures requiring physician visits and diagnosis were included, and since follow-up visits were at very frequent (on average 4-mo) intervals, it is unlikely that fractures were missed.

Fractures resulting from automobile accidents were excluded from the analyses because the force of trauma was considered to be an overwhelming factor. Spinal fractures were also excluded since a systematic effort to look for asymptomatic or undiagnosed fractures in the spine was not made. Routine lateral $x$-rays would have been required on all subjects at regular intervals to assess the incidence of crush fractures and this was considered unreasonable, given the long followup. In addition to analyzing all other fractures, a specific study of hip and wrist fractures was made for comparison with other studies. Hip fractures were identified as fractures at the neck or trochanter of the femur. Since Colles' fractures could not be reliably identified in some of the younger subjects, a subgroup of fractures of the distal forearm that included distal radius, distal ulna, and wrist were studied. In addition, other fractures were studied as two subgroups: (a) upper forearm, leg (tibia, fibula, and shaft of femur), ankle, and pelvis, the 
incidence of which increases with age; and $(b)$ skull, hands, and feet, the incidence of which does not increase with age according to previous studies (7-12).

Data reduction. The periods of follow-up were divided into intervals according to the subject's age at the time of each bone measurement: younger than age 45, 5-yr intervals from age 45 to 79 , and older than age 79. For example, a subject who was followed from age 58 to 73 and measured yearly would contribute 2 person-yr to the 55-59-yr interval, 5 person-yr each to the 60-64- and 65-69-intervals, and 3 person-yr to the 70-74-yr interval. For each interval in each subject, the mean bone mass of all bone measurements made within that interval was calculated. Each interval of a subject period was then classified by the mean bone mass at increments of $0.1 \mathrm{~g} / \mathrm{cm}$. Thus, each subject was periodically reclassified by age interval and mean bone mass. For each age-by-mean bone-mass category, the total of personyears that were contributed by all subjects was calculated. Fractures were assigned to the age interval and bone-mass increment of the individual at the time of fracture, and fractures per person-year for each age- and bone-mass-interval were obtained.

Statistical analysis. Within each age interval of each subject, fractures were assumed to occur randomly over time with an underlying rate determined by the person's age and level of bone mass. From the assumption of a random occurrence of fractures, the number of fractures in each age interval for an individual with a given bone mass would be expected to follow a Poisson distribution and to have a certain mean which would be equal to the variance. However, when data were combined from different individuals with the same bone mass in the same age interval, we expected the Poisson variance to be inflated by a factor due to between-subject variability. In other words, the variance of the total number of fractures of all subjects in an interval would be greater than the expected total number of fractures. Therefore, in the statistical model we allowed a scale factor to reflect the extra-Poisson variance. Since we no longer had an exact Poisson distribution we used a quasi-likelihood approach for parameter estimation (13), which depends only on the algebraic form of the mean and variance and does not require the specification of the exact distribution. In our model, we assumed that the variance was proportional to the mean. Using this technique, we estimated the fracture rate in each category as a function of age and bone mass in a log-linear model. The scale-factor that reflected the extra-Poisson variance was then estimated from a Pearson's chi-square statistic calculated from the residuals of the fitted model. If the scale-factor was greater than one, it was used to adjust the variance estimates and the test statistics. The Pearson's chi-square statistic could also be interpreted as a goodnessof-fit test for the exact Poisson model (i.e., if the scale factor equaled one). This particular statistical approach was chosen because it was appropriate for analyzing categorical data for which some subjects had contributed data to more than one category. An outline of the general approach is given in the appendix. The statistical modeling was performed using generalized linear interactive modeling (14).

\section{Results}

The 521 subjects contributed a total of 3,388 person-yr of follow-up. The distributions of their age at entry, the length of follow-up, and the number of bone-mass measurements are given in Table I.

The first set of analyses was based on a total of 138 fractures at all sites; the distribution of sites is shown in Table II. Hip fractures occurred most frequently $(n=34)$ and face/skull fractures least frequently $(n=2)$. Table III lists the number of person-years at risk and the number of observed and expected fractures for each age and bone-mass interval. The logarithm of the rate of fracture was fitted to a linear model of age and bone mass. The results showed that the linear terms of both factors were significant $(P<0.05)$, but that their quadratic
Table I. Characteristics of 521 Caucasian Women Studied

\begin{tabular}{lrrcc}
\hline & Mean & SD & Minimum & Maximum \\
\hline Age at entry $(y r)$ & 57.4 & 16.8 & 22.7 & 95.8 \\
Length of follow-up $(y r)$ & 6.5 & 4.0 & 1.0 & 15.0 \\
$\begin{array}{l}\text { Number of bone-mass } \\
\quad \text { measurements }\end{array}$ & 24.6 & 16.5 & 2 & 74 \\
\hline
\end{tabular}

terms and the interaction between age and bone mass were not. The fitted model is given by:

expected number of fractures per person-year

$=\exp [-5.883-2.014$ (bone mass) +0.06114 (age) $]$,

where bone mass is in grams per centimeter and age is in years.

The goodness-of-fit statistic from the observed and expected cell frequencies equaled 48.4 for a chi-square distribution with 45 degrees of freedom. (Under the Poisson model, the expected value of chi-square statistic equals the number of degrees of freedom. If the chi-square statistic is nearly equal to or smaller than the degree of freedom, the data are consistent with an exact Poisson model.) The plot of the standardized residuals displayed a near-normal distribution. These results showed that the log-linear model was consistent with the assumption that fractures occur randomly at a rate determined by age and bone mass alone, and that no additional betweensubject variability contributed substantially to the rate. Thus, it was appropriate to assume that the scale factor in the model was equal to one and that no adjustments were necessary for the variance estimates or for the test statistics.

The fracture risk from the fitted model is plotted against bone mass for various age groups, and against age for various levels of bone mass in Fig. 1. From Fig. $1 A$ we see that for every age group the relative risk of fracture between the lowest and the highest level of bone mass is greater than two, but because the rate of fracture increases significantly with age, the absolute increase in fracture risk for a given deficit in bone mass is much greater for older than for younger women. Within the range of age and bone mass observed in this study, age appeared to have a stronger effect on fracture rate. This is evidenced by the steeper rise in fracture rate with age for a given level of bone mass (Fig. $1 B$ ) compared with the rise of

Table II. Distribution of Site of 138 Fractures Experienced by 521 Study Subjects

$\begin{array}{lr}\text { Face/skull } & 2 \\ \text { Rib/clavicle } & 23 \\ \text { Upper arm } & 9 \\ \text { Radius/ulna } & 12 \\ \text { Wrist } & 5 \\ \text { Hand/finger } & 11 \\ \text { Pelvis } & 5 \\ \text { Hip } & 34 \\ \text { Leg (long bones) } & 14 \\ \text { Patella } & 3 \\ \text { Foot } & 17 \\ \text { Ankle } & 3\end{array}$


Table III. Number of Fractures Per Person-Years at Risk by Age and Bone-Mass Intervals (Expected Values in Parentheses)

\begin{tabular}{|c|c|c|c|c|c|c|c|}
\hline \multirow[b]{2}{*}{ Age (yr) } & \multicolumn{7}{|c|}{ Radius midshaft bone mass $(\mathrm{g} / \mathrm{cm})$} \\
\hline & $<0.60$ & $0.60-0.69$ & $0.70-0.79$ & $0.80-0.89$ & $0.90-0.99$ & $1.0+$ & Total \\
\hline$<45$ & - & $\begin{array}{c}0 / 6.3 \\
(0.1)\end{array}$ & $\begin{array}{r}0 / 43.3 \\
(0.4)\end{array}$ & $\begin{array}{c}0 / 134.3 \\
(0.9)\end{array}$ & $\begin{array}{c}1 / 135.0 \\
(0.7)\end{array}$ & $\begin{array}{c}0 / 107.4 \\
(0.5)\end{array}$ & $\begin{array}{c}1 / 426.3 \\
(2.6)\end{array}$ \\
\hline $45-49$ & - & $\begin{array}{c}0 / 2.2 \\
(0)\end{array}$ & $\begin{array}{r}0 / 44.9 \\
(0.5)\end{array}$ & $\begin{array}{c}2 / 131.2 \\
(1.2)\end{array}$ & $\begin{array}{c}2 / 107.6 \\
(0.8)\end{array}$ & $\begin{array}{r}0 / 49.3 \\
(0.3)\end{array}$ & $\begin{array}{c}4 / 335.2 \\
(2.8)\end{array}$ \\
\hline $50-54$ & - & $\begin{array}{r}1 / 19.6 \\
(0.4)\end{array}$ & $\begin{array}{c}0 / 106.5 \\
(1.6)\end{array}$ & $\begin{array}{c}3 / 118.0 \\
(1.5)\end{array}$ & $\begin{array}{c}1 / 115.8 \\
(1.2)\end{array}$ & $\begin{array}{r}0 / 39.0 \\
(0.3)\end{array}$ & $\begin{array}{c}5 / 398.9 \\
(5.0)\end{array}$ \\
\hline $55-59$ & $\begin{array}{c}0 / 5.4 \\
(0.2)\end{array}$ & $\begin{array}{r}3 / 63.3 \\
(1.6)\end{array}$ & $\begin{array}{c}7 / 139.2 \\
(2.9)\end{array}$ & $\begin{array}{c}5 / 156.0 \\
(2.6)\end{array}$ & $\begin{array}{r}1 / 95.0 \\
(1.3)\end{array}$ & $\begin{array}{r}0 / 35.6 \\
(0.4)\end{array}$ & $\begin{array}{c}16 / 494.5 \\
(9.0)\end{array}$ \\
\hline $60-64$ & $\begin{array}{r}1 / 41.7 \\
(1.8)\end{array}$ & $\begin{array}{r}2 / 87.8 \\
(3.0)\end{array}$ & $\begin{array}{c}4 / 159.4 \\
(4.5)\end{array}$ & $\begin{array}{c}4 / 152.6 \\
(3.5)\end{array}$ & $\begin{array}{r}0 / 32.4 \\
(0.6)\end{array}$ & $\begin{array}{r}0 / 22.6 \\
(0.3)\end{array}$ & $\begin{array}{c}11 / 496.5 \\
(13.7)\end{array}$ \\
\hline $65-69$ & $\begin{array}{r}4 / 56.1 \\
(3.2)\end{array}$ & $\begin{array}{r}0 / 91.0 \\
(4.2)\end{array}$ & $\begin{array}{c}6 / 109.5 \\
(4.2)\end{array}$ & $\begin{array}{r}0 / 45.6 \\
(1.4)\end{array}$ & $\begin{array}{r}0 / 15.4 \\
(0.4)\end{array}$ & $\begin{array}{r}0 / 6.3 \\
(0.1)\end{array}$ & $\begin{array}{c}10 / 323.9 \\
(13.6)\end{array}$ \\
\hline $70-74$ & $\begin{array}{r}6 / 56.0 \\
(4.3)\end{array}$ & $\begin{array}{r}4 / 69.2 \\
(4.4)\end{array}$ & $\begin{array}{r}1 / 63.0 \\
(3.3)\end{array}$ & $\begin{array}{c}0 / 8.2 \\
(0.3)\end{array}$ & $\begin{array}{r}0 / 4.7 \\
(0.2)\end{array}$ & - & $\begin{array}{c}11 / 201.1 \\
(12.5)\end{array}$ \\
\hline $75-79$ & $\begin{array}{r}2 / 47.7 \\
(5.0)\end{array}$ & $\begin{array}{r}3 / 47.3 \\
(4.1)\end{array}$ & $\begin{array}{r}2 / 43.7 \\
(3.1)\end{array}$ & $\begin{array}{r}0 / 18.0 \\
(1.0)\end{array}$ & $\begin{array}{c}0 / 3.3 \\
(0.2)\end{array}$ & - & $\begin{array}{l}7 / 160.0 \\
(13.3)\end{array}$ \\
\hline $80+$ & $\begin{array}{c}34 / 208.6 \\
(29.8)\end{array}$ & $\begin{array}{c}12 / 167.5 \\
(19.5)\end{array}$ & $\begin{array}{c}26 / 151.6 \\
(14.5)\end{array}$ & $\begin{array}{r}1 / 12.9 \\
(1.0)\end{array}$ & $\begin{array}{r}0 / 11.9 \\
(0.8)\end{array}$ & - & $\begin{array}{c}73 / 552.5 \\
(65.5)\end{array}$ \\
\hline Total & $\begin{array}{c}47 / 415.5 \\
(44.3)\end{array}$ & $\begin{array}{c}25 / 554.2 \\
(37.3)\end{array}$ & $\begin{array}{c}46 / 861.1 \\
(34.8)\end{array}$ & $\begin{array}{c}15 / 776.8 \\
(13.5)\end{array}$ & $\begin{array}{c}5 / 521.1 \\
(6.1)\end{array}$ & $\begin{array}{c}0 / 260.2 \\
(2.0)\end{array}$ & \\
\hline
\end{tabular}

fracture rate with bone mass at any given age (Fig. $1 A$ ). While this of course reflects the scales used for the plots, these plots describe the fracture risk only across the observed range of data. For example, a difference of $0.1 \mathrm{~g} / \mathrm{cm}$ of bone mass (at age 65-69) confers a difference in fracture risk of $8 / 1,000$ person-yr; a difference in age of $10 \mathrm{yr}$ (at a bone mass of $0.8-0.89$ ) confers a difference in fracture risk of $21 / 1,000$ person-yr. No comparison of this type can be perfectly fair, but a $0.1 \mathrm{~g} / \mathrm{cm}$ decline in bone mass approximates the average population change in $10 \mathrm{yr}(1 \% / \mathrm{yr})$, and thus the two and a half times greater risk for this difference in age, compared with the difference in bone mass, does reflect the relative strength of these risk factors.

We also examined the distribution of the 34 hip fractures.

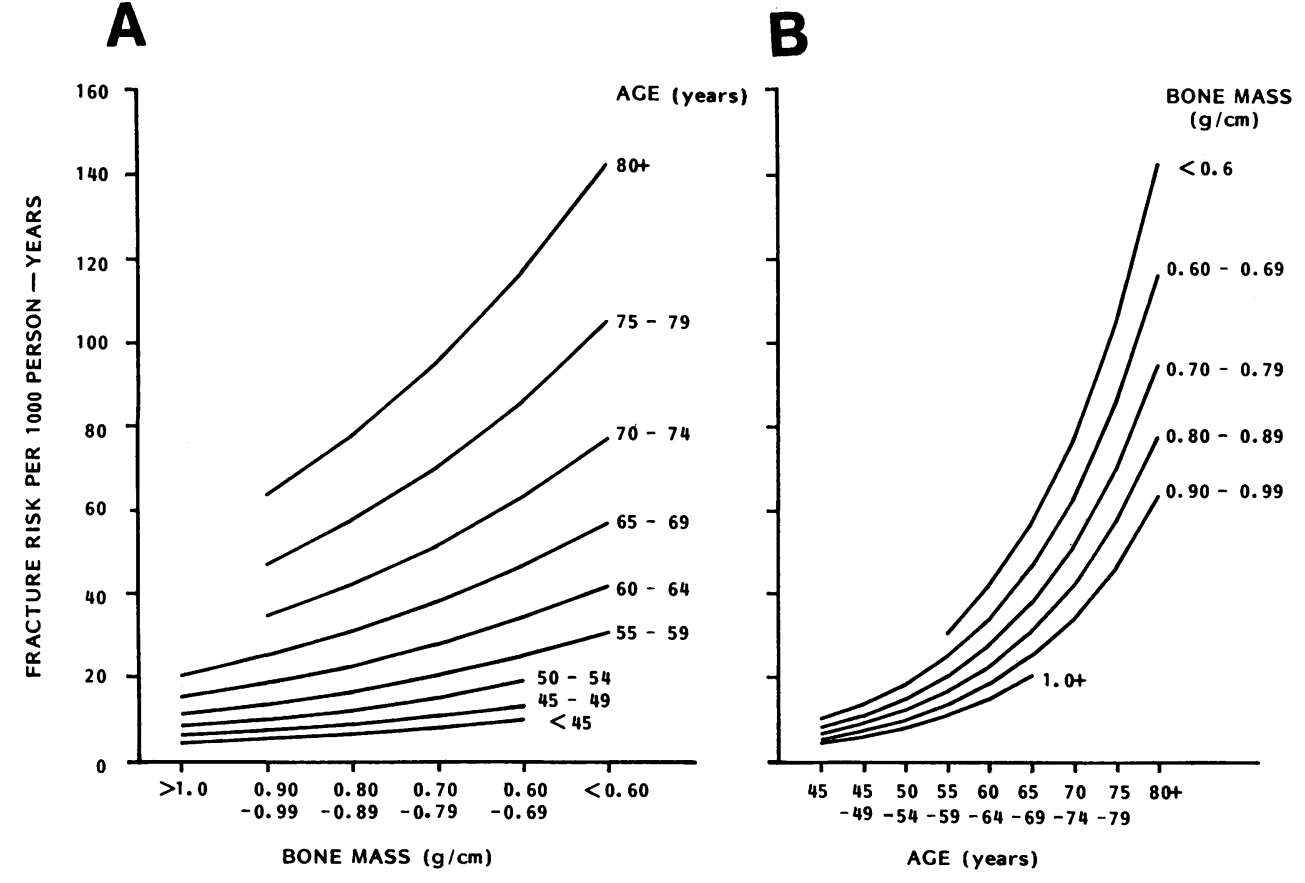

Figure 1. Estimated incidence of fracture as a function of age and bone mass. 
Table IV. Observed and Fitted* Frequencies and Rates of Hip Fractures by Age and Bone-Mass Intervals

\begin{tabular}{|c|c|c|c|c|}
\hline \multirow[b]{2}{*}{ Age (yr) } & \multicolumn{3}{|c|}{ Radius midshaft bone mass $(\mathrm{g} / \mathrm{cm})$} & \multirow{2}{*}{$\begin{array}{c}\text { Rate per } 1,000 \\
\text { person-yr }\end{array}$} \\
\hline & 0.7 & $0.7-0.89$ & $0.9+$ & \\
\hline \multirow[t]{2}{*}{$<50$} & 0 & 0 & 0 & 0 \\
\hline & $(0.00)$ & $(0.08)$ & $(0.10)$ & $(0.24)$ \\
\hline \multirow[t]{2}{*}{$50-59$} & 0 & 1 & 0 & 1.12 \\
\hline & $(0.08)$ & $(0.46)$ & $(0.25)$ & $(0.89)$ \\
\hline \multirow[t]{2}{*}{$60-69$} & 0 & 2 & 0 & 2.44 \\
\hline & $(0.92)$ & $(1.54)$ & $(0.26)$ & $(3.32)$ \\
\hline \multirow[t]{2}{*}{$70-79$} & 4 & 2 & 0 & 16.62 \\
\hline & $(2.74)$ & $(1.66)$ & $(0.10)$ & $(12.45)$ \\
\hline \multirow[t]{2}{*}{$80+$} & 20 & 5 & 0 & 45.25 \\
\hline & $(17.58)$ & (7.69) & $(0.56)$ & $(46.70)$ \\
\hline
\end{tabular}

* All fitted values are in parentheses.

Since the original classification of age and bone mass yielded a very sparse table, we combined the levels of age and bone mass into broader categories. The observed frequencies are shown in Table IV. When a log-linear model was fitted to the rate, bone mass was no longer a significant predictor once age was in the model. The final model was:

expected number of hip fractures per person-year

$=\exp [-14.3+0.132$ (age) $]$.

The chi-square goodness-of-fit statistic was 5.05 with 13 degrees of freedom. The observed and expected frequencies of hip fractures (see Table IV) are consistent with the assumptions of a Poisson model. The observed and expected rates of hip fractures for different age groups are also shown in Table IV. It should be noted that when both age and bone mass were included in the model for hip fractures, the regression coefficient $(-2.3)$ for bone mass was almost identical to its corresponding value in the model for predicting all fractures (see Eq. 1). The regression coefficient was not significantly different from zero in the hip fracture model because of the smaller number of outcomes.

We further examined the distribution of 17 fractures at the distal forearm. Even broader categories of age and bone mass were used because of the small number of fractures. The frequency distribution is shown in Table $\mathrm{V}$. When the rate of fracture was fitted to a log-linear model, bone mass alone resulted in a better fit than did age alone, and the use of both variables did not improve the fit of the model significantly. The fitted model simply states that for bone mass $>0.8 \mathrm{~g} / \mathrm{cm}$, the risk of fracture at the distal forearm is $0.64 / 1,000$ person-yr and that the risk increases to $8.74 / 1,000$ person-yr when bone mass drops below $0.8 \mathrm{~g} / \mathrm{cm}$.

At the distal forearm, the expected frequency of fracture for each level of bone mass can be compared with the corresponding observed frequency in Table V. The chi-square goodness-of-fit statistic was 4.02 with 4 degrees-of-freedom, again consistent with a Poisson distribution.

The results from the analysis of fractures, which consisted of upper forearm, legs, ankles, and pelvis, were similar to the results for fractures at the distal forearm, i.e., bone mass alone
Table V. Observed and Fitted ${ }^{*}$ Frequencies and Rates of Fractures at the Distal Forearm by Age and Bone-Mass Intervals

\begin{tabular}{ccc}
\hline & \multicolumn{2}{c}{ Radius midshaft bone } \\
& mass $(\mathrm{g} / \mathrm{cm})$ & $0.8+$ \\
\cline { 2 - 3 } Age (yr) & $\leqslant 0.8$ & 0 \\
555 & 0 & $(0)$ \\
$55-69$ & $(0)$ & 1 \\
& 7 & $(0.16)$ \\
$70+$ & $(6.59)$ & 0 \\
& 9 & $(0.04)$ \\
\hline Rate per 1,000 & $(7.47)$ & 0.64 \\
person-yr & 8.74 & $(0.64)$ \\
\hline
\end{tabular}

* All fitted values are in parentheses.

was a slightly better predictor of fracture incidence than age alone, but inclusion of both predictors did not improve the fit of the model. The regression coefficient $(-2.4)$ for bone mass was comparable to the corresponding regression coefficient $(-2.0)$ in predicting all fractures (see Eq. 1).

For the group of fractures (skull, hands, and feet) not previously found to increase with age, we did find an increasing incidence with age, although the number of fractures was quite small. Bone mass alone was a much weaker predictor of these fractures. Furthermore, forcing both age and bone mass into the model led to a positive but insignificant regression coefficient for bone mass, indicating that bone mass has no additional predictive value beyond age for this group of fractures.

\section{Discussion}

From this study, we found that the rate of all nonspinal fractures increases with decreasing bone mass and with increasing age. This is the first time that these two factors have been shown directly to have independent effects on fracture risk, although this finding was not surprising based on the indirect evidence from previous studies.

Most studies of bone mass and fracture risk in living human subjects have been based on the comparison of bone mass of subjects who have or have not sustained fractures in the past. Although these studies usually find a significantly lower mean bone mass in the fracture group, the overlap between the cases and controls is always large, which has led some researchers to conclude that bone mass is not useful in identifying subjects at risk of fracture (3). Studies using agematched controls in particular have found only small differences because almost all women lose bone with age and most of the fractures occur at older ages. Because of all the problems mentioned above, and the usual biases associated with retrospective studies, prospective studies provide a more valid assessment of the role of bone mass in predicting fractures. More importantly, prospective studies give true estimates of relative risks without the assumption of rare outcomes when fractures may not be such rare events in the elderly.

The few published prospective studies of the effects of bone 
mass on fracture risk have some methodologic problems. Smith et al. (4) did not account for the effects of age, which is highly correlated with bone mass; they also ignore the variable lengths of follow-up of the subjects. The findings of Wasnich et al. (5) are based on very small numbers of fractures with a relatively large number of covariates so that their estimates of relative risks must have large SEs, although these were not reported.

From the previous studies, we decided that the best way to assess the role of bone mass in fracture risk was to conduct a prospective study using absolute bone mass rather than relative bone mass for a given age, and to assess simultaneously the effect of age as an independent predictor. Our results showed the additivity of the effects of age and bone mass on the logarithm of fracture rate. Thus, their effects on the actual rate of fracture are multiplicative; i.e., the increase in fracture rate due to a given amount of bone mass deficit becomes greater as a subject gets older. Since we included all nonspinal fractures, it is possible that fractures at some sites may not be related to osteoporosis. The inclusion of such fractures could have weakened the relationship between bone mass and fractures, but we chose the more objective estimate of the risk of all fractures without qualification.

The usefulness of bone mass in predicting fractures persisted when we examined the subgroup of fractures at the distal forearm. Bone-mass measurements were stronger than age in predicting these fractures. This agrees with Melton's explanation of the plateau of incidence rates of Colles' fracture with slowing bone loss later in life (2).

From reviewing the indirect evidence of previous studies, Cummings (3) has concluded that bone-mass measurements do not predict hip fractures. We now have direct evidence showing that age is a much stronger predictor of hip fractures than is radius bone mass. This finding, however, is not different from that for all fractures combined. Indeed, when age and bone mass are both included in the model for predicting hip fracture, the estimated relative risk due to decreasing bone mass is almost identical to its corresponding estimate for all fractures. However, because of the smaller number of hip fractures in this study, this magnitude of relative risk is no longer statistically significant. Although Melton et al. (15) concluded that bone-mass measurements at the hip were predictive of the risk of hip fracture, their findings are based on indirect evidence from both the population age-distribution of hip fractures and the age-specific distribution of bone measurements from a small survey. Briefly, an age-stratified random population sample of all women aged 35 and over was selected for bone-mass measurements of the hip, and the distribution of bone density was estimated as a function of age. A normal distribution of bone density was also fitted to similar measurements on 49 hip fracture patients. The incidence rate of hip fractures for any specified range of bone density was then calculated from the expected numbers of fractures and women at risk derived from these fitted distributions. The value of bone-mass measurements at the hip in predicting hip fractures remains to be assessed from direct prospective studies.

Bone mass was also found to predict the risk of most other fractures except at the skull, hands, and feet. Our results are consistent with the findings from many epidemiologic studies of site-specific fractures at Mayo Clinic (7-12). They have also suggested that, except for skulls, hands, and feet, most frac- tures are related to bone mass because of their increasing incidence with age.

We measured bone mass at the radius instead of other sites purely for historical reasons. When the women were first studied in 1971, this was the only technique available for repeated measurements that did not pose excessive radiation problems. To take advantage of the long-term follow-up, we have continued to measure these women at the radius. However, photon densitometry measurements at other sites have since become available and it is possible that measurements at other sites will be even stronger predictors of fractures, especially those at the site of fracture.

The magnitude of relative risks between different levels of bone mass is similar to those for many risk factors for other diseases. Fracture is as prevalent in older women as coronary heart disease (CHD) ${ }^{1}$ is in men. Thus, the attributable risk due to low bone mass (the difference in rates of fractures between high and low bone-mass groups) is at least as large as the attributable risk for factors associated with other diseases. To give perspective on the usefulness of bone measurements for predicting fractures, we quote some estimates for the use of total serum cholesterol in predicting CHD. In the Framingham study, the 24-yr incidence of CHD for men starting at age $55-59$ was $604 / 1,000(16)$. The relative risk (estimated by relative odds) of CHD is 1.25 for every SD increase in cholesterol (17). For women, starting at age 60, the 24-yr incidence of fractures is $794 / 1,000$ from this study, and the relative risk is 1.3 for every SD of decrease in bone mass. From the order of magnitude of these estimates, we can conclude that bone mass is at least as useful in predicting fractures as cholesterol is in predicting CHD.

The strong effect of age on the risk of fracture does not mean that fractures should be accepted as part of the aging process. Since we drew most of our older women from a retirement home, they may have been more uniformly debilitated than the "free-living" elderly. This may be the reason we did not find large between-subject variances after accounting for age and bone mass. However, aging itself is probably not a direct risk factor but the physiologic changes associated with it must account for the increase in the number of fractures. Further research is needed to identify these factors.

\section{Appendix}

If fractures occur randomly over time at some underlying mean rate, it is said to follow a Poisson process. For an individual with bone mass $x_{1}$ at age $x_{2}$, the probability of sustaining $k$ fractures in a unit time is given by

$\operatorname{Pr}(Y=k)=\frac{\mathrm{e}^{-\lambda}(\lambda)^{k}}{k !}$,

where $\lambda$ is the underlying rate of fracture and $\operatorname{Var}(Y)=E(Y)$.

The rate $\lambda$ can be modeled as a linear function of bone mass and age:

$\log \lambda=\beta_{0}+\beta_{1} x_{1}+\beta_{2} x_{2}$.

It follows that for a number of individuals contributing a total of $N$ person-years at risk,

$\log$ (expected number of fractures) $=\ln N+\beta_{0}+\beta_{1} x_{1}+\beta_{2} x_{2}$.

1. Abbreviations used in this paper: $\mathrm{CHD}$, coronary heart disease. 
If there is inter-subject variability in fracture risk other than that attributable to age and bone mass, we expect

$\operatorname{var}(Y)=\sigma^{2} E(Y)$,

where $\sigma^{2}$ is a scale-factor $>1$.

With the mean and variance defined, we may use the quasi-likelihood approach to estimate the model parameters (6). The estimation of $\left(\beta_{0}, \beta_{1}, \beta_{2}\right)$ is independent of $\sigma^{2}$, so algorithms for log-linear models assuming Poisson distributions can be applied. The scale-factor $\sigma^{2}$ can then be estimated from the residuals of the fitted model:

$\hat{\sigma}^{2}=\sum \frac{(O-E)^{2}}{E} /(n-p)$,

where $O$ and $E$ are, respectively, the observed and expected (from fitted model) number of fractures in each age-by-bone mass combination, $n$ is the total number of such combinations, and $p$ is the number of unknown parameters other than $\sigma^{2}$. If $\hat{\sigma}^{2}>1$, variance estimates from fitting the Poisson models are multiplied by $\hat{\sigma}^{2}$ and SEs are multiplied by $\hat{\sigma}$. All hypothesis testing are based on the inflated variance estimates.

\section{Acknowledgments}

This research was partially supported by a Research Career Development Award to Dr. Hui, National Institutes of Health grants RO1 AG-4518 and PO1 AG-05793, and a grant from Ancient and Accepted Scottish Rite, Valley of Indianapolis.

\section{References}

1. Mazess, R. B. 1982. On aging bone loss. Clin. Orthop. Relat. Res. 162:239-252.

2. Melton, L. J., III, E. Y. S. Chao, and J. Lane. 1988. Biomechanical aspects of fractures. In Osteoporosis: Etiology, Diagnosis and Management. B. L. Riggs and L. J. Melton, editors. Raven Press, New York. 111-131.

3. Cummings, S. R. 1985. Are patients with hip fractures more osteoporotic? Am. J. Med. 78:487-494.

4. Smith, D. M., M. R. A. Khairi, and C. C. Johnston, Jr. 1975. The loss of bone mineral and its relationship to risk of fracture. J. Clin. Invest. 56:311-318.
5. Wasnich, R. D., P. D. Ross, L. K. Heilbrun, and J. M. Vogel. 1987. Selection of the optimal skeletal site for fracture risk prediction. Clin. Orthop. Relat. Res. 216:262-268.

6. Slemenda, C., S. L. Hui, C. Longcope, and C. C. Johnston, Jr. 1987. Sex steroids and bone mass. A study of changes about the time of menopause. J. Clin. Invest. 80:1261-1269.

7. Rose, S. H., L. J. Melton III, B. F. Morrey, D. M. Ilstrup, and B. L. Riggs. 1982. Epidemiologic features of humeral fractures. Clin. Orthop. Relat. Res. 168:24-30.

8. Owen, R. A., L. J. Melton III, K. A. Johnson, D. M. Ilstrup, and B. L. Riggs. 1982. Incidence of Colles' fracture in a North American community. Am. J. Public Health. 72:605-607.

9. Daly, P. J., R. H. Fitzgerald, Jr., L. J. Melton III, and D. M. Ilstrup. 1987. Ankle fractures in Rochester, MN, 1979-81. Acta Orthop. Scand. 58:539-544.

10. Melton, L. J. III, J. M. Sampson, B. F. Morrey, and D. M. Ilstrup. 1981. Epidemiologic features of pelvic fractures. Clin. Orthop. Relat. Res. 155:43-47.

11. Arneson, T. J., L. J. Melton III, D. G. Lewallen, and W. M. O'Fallon. 1987. Epidemiology of diaphyseal and distal femur fractures in Rochester, Minnesota, 1965-1984. Clin. Orthop. Relat. Res. In press.

12. Nelson, E. L., L. J. Melton III, J. F. Annegers, E. R. Laws, and K. P. Offord. 1984. Incidence of skull fractures in Olmsted County, Minnesota. Neurosurgery (Baltimore). 15:318-324.

13. McCullagh, P., and J. A. Nelder. 1983. Generalized Linear Models. Chapman and Hall, London/New York.

14. Baker, R. J., and Nelder, J. A. 1978. The GLIM System, Release 3, Generalized Linear Interactive Modelling. Numerical Algorithms Group, Oxford.

15. Melton, L. J., H. W. Wahner, L. S. Richelson, W. M. O'Fallon, and B. L. Riggs. 1986. Osteoporosis and the risk of hip fracture. Am.J. Epidemiol. 124:254-261.

16. Gordon, T., P. Sorlie, and W. B. Kannel. 1971. The Framingham Study. An Epidemiological Investigation of Cardiovascular Disease. Section 27. Coronary Heart Disease, Atherothrombotic Brain Infarction, Intermittent Claudication: A Multivariate Analysis of Some Factors Related to Their Incidence. Framingham Study, 16-year Follow-up. U. S. Department of Health, Education, and Welfare.

17. Gordon, T., and W. B. Kannel. 1972. Predisposition to atherosclerosis in the head, heart, and legs: the Framingham study. JAMA (J. Am. Med. Assoc.) 221:661-666. 